\title{
EXPERIMENTAL MULTI-LEVEL SEISMIC PERFORMANCE ASSESSMENT OF 3D RC FRAME DESIGNED FOR DAMAGE AVOIDANCE
}

\author{
Brendon A Bradley ${ }^{1 *}$, Rajesh P Dhakal ${ }^{1}$, John B Mander ${ }^{1}$, Louman Li $^{1}$. \\ ${ }^{1}$ Department of Civil Engineering, University of Canterbury, Private Bag 4800, Christchurch 8020, New Zealand \\ *Corrosponding author: $\mathrm{Ph}+64-3-3642987$ ext 7333; Fax: +64-3-364 2758; \\ Email: bab54@student.canterbury.ac.nz
}

\begin{abstract}
This paper experimentally investigates the application of damage avoidance design (DAD) philosophy to moment resisting frames with particular emphasis on detailing of rocking interfaces. An $80 \%$ scale 3-dimensional rocking beam-column joint sub-assembly designed and detailed based on damage avoidance principles is constructed and tested. Incremental dynamic analysis (IDA) is used to select ground motion records to be applied to the sub-assembly to conduct a multi-level seismic performance assessment (MSPA). Analyses are conducted to obtain displacement demands due to the selected near and medium field ground motions that represent different levels of seismic hazard. Thus predicted displacement time histories are applied to the sub-assembly to conduct quasi-earthquake displacement (QED) tests. The sub-assembly performed well reaching drifts up to $4.7 \%$ with only minor spalling occurring at rocking beam interfaces and minor flexural cracks in beams. Yielding of post-tensioning tendons occurred, but the sub-assembly did not collapse. The externally attached energy dissipaters provided large hysteretic dissipation during large drift cycles. The sub-assembly satisfied all three seismic performance requirements, thereby verifying the superior performance of the DAD philosophy.
\end{abstract}




\section{KEYWORDS}

multi-level seismic performance assessment; damage avoidance design; incremental dynamic analysis; quasi-earthquake displacement tests.

\section{INTRODUCTION}

Currently, seismic design of structures are performed using capacity design philosophy, which aims to prevent collapse by restricting inelastic behaviour in well-detailed plastic hinge regions that have adequate ductility capacity to sustain inelastic displacements imposed during an earthquake. During recent earthquakes such as Loma Prieta (1989), Northridge (1994) and Kobe (1995), structures performed as they were designed, with few collapses. However, the economic losses in many cases were extensive, not only due to disruption in the functionality of those buildings which were no longer able to be occupied immediately, but also due to significant cost incurred in repairing the damages sustained by structures while undergoing large inelastic deformation to meet the seismic demands. In the aftermath of these events came a higher demand from clients and the public for engineers to produce structural systems which not only ensure life safety but also avoid severe structural damage during earthquakes. Damage avoidance design (DAD) is an approach which has shown promise of fulfilling this objective. DAD aims to accommodate the seismic displacement demands on structures through rocking mechanisms at the interfaces between different members without incurring any damage on the members themselves.

The rocking motion mechanism was first studied by Housner [1] who developed an expression that fully describes the amplitude dependent rocking motion of a rigid block in terms of its weight, mass moment of inertia about its rocking toe and radius to the centre of gravity. It was not until some years later that investigation into the performance of frame-like structures with rocking connections was commenced. A multi-phase research programme 
was initiated at the National Institute of Standards and Technology (NIST) in 1987. The main objective was to develop guidelines for an economical precast beam-to-column connection for regions of high seismic activity. In the first phase of this research programme, Cheok and Lew [2] tested four monolithic and two equivalent post tensioned precast concrete connections. From their results, they showed that post-tensioned precast concrete beamcolumn connections were as strong and ductile as monolithic connections and the precast connection was therefore a viable option for high seismic regions. However, the posttensioned precast concrete beam-column connection exhibited much lesser energy dissipation capacity than that of monolithic specimens. Several methods of increasing energy dissipation of precast concrete connection were explored in the second and third phases of the NIST programme [3]. It was found that after moderate ductility levels had been achieved, the test specimens suffered excessive loss of stiffness. This loss of stiffness was thought to be caused by a reduction in effective prestressing force through the column, resulting from inelastic strain of the prestressing tendon.

Priestley and Tao [4] proposed a concept using partially-unbonded prestressing tendons through rocking beam-column connections to explicitly provide the restoring force. Several nonlinear dynamic analyses were carried out on single degree of freedom oscillators of different initial natural periods and hysteretic characteristics which represented partially bonded jointed construction and monolithic construction. They concluded that for longer period structures ( $\mathrm{T}>1.2$ seconds) the displacement demand for partially prestressed systems characterised by a bilinear elastic hysteretic response was similar to that for equivalent monolithic construction. In the last phase of the NIST programme, a total of 10 so-called "hybrid" precast connections with different combinations of mild steel and post-tensioning tendons were tested [5]. It was found that a hybrid precast system can be designed to have a flexural strength similar to a conventionally designed monolithic system. Yet the hybrid 
system will suffer less damage, exhibit reasonable energy dissipation capability, and undergo large drifts while still maintaining a re-centring capability.

A US-Japan cooperative research programme on precast seismic structural systems (PRESSS) [6] performed extensive investigation into jointed unbonded post-tensioned beamcolumn connections. The programme concluded with a test on a five-storey precast concrete building comprised of four different structural frame systems in one direction and a hybrid structural wall system in the orthogonal direction. Both frames and wall performed well with much less damage than equivalent monolithic frame and walls when subjected to the same drift levels. Little residual displacement was observed in both frames and walls.

Mander and Cheng [7] proposed a design method based on damage avoidance principles (referred to as DAD hereafter), in which jointed members are armoured at their ends and protected from being damages. Some energy dissipation is permitted to occur within replaceable "fuse" components of the structure. Mander and Cheng [7] then investigated the performance of bridge piers designed to rock at the top and bottom of pier columns under lateral loading. A complete force-displacement model of the rocking system was developed considering pre-rocking flexibility, rigid body kinematics and accounting for the effect of post-tensioning the pier to the foundation. Their findings showed that the steel armouring at pier end zones effectively prevented damage to the rocking connection at the top and bottom of the pier. Previous studies using the DAD philosophy have included bridge piers [8], walls [9-10] and beam-column joints [11-12]. However, the DAD beam-column drift tests used quasi-static loading which is composed of regulated displacement cycles. These regulated displacement cycles are however not completely representative of the displacement demands due to seismic loading.

In this study an experimental investigation is conducted on a 3-dimensional large scale beam-column joint from a typical New Zealand moment resisting frame building using the 
actual irregular displacement history more likely to be experienced during an earthquake. A multi-level seismic performance assessment (MSPA) methodology is established and applied to the building by using pre-identified critical ground motion records representative of different levels of seismic hazard [13]. By applying these critical ground motion records to computational models of the prototype building, displacement-time histories of certain elements of the building are extracted. These displacement-time histories are then applied to the beam-column joint sub-assembly in quasi-earthquake displacement (QED) tests.

\section{EXPERIMENTAL INVESTIGATION}

\section{Specimen Details/Design}

The 3D beam-column joint specimen tested in this study is an exterior joint on the second floor of a ten-storey reinforced concrete frame building. The original $3 \times 3$ bays moment resisting frame building, which is well-documented as the Red Book Building [14], was designed according to the New Zealand Standard, NZS3101 [15]. Keeping all other design variables constant, the same building was designed and detailed according to the DAD principles, thereby resulting in the precast and prestressed beam and columns being connected to each other at steel armoured rocking interfaces. The DAD building had precast flooring units running along the seismic-frame direction and resting on the gravity-beams at the two ends. Columns were precast with rocking interfaces at the ground floor and underside of the $6^{\text {th }}$ floor. Beam-column connections on the first five floors comprise rocking connections with supplemental external energy dissipators. Beam-column joints above the $6^{\text {th }}$ floor have monolithic connections. The $80 \%$ scaled 3-dimensional beam-column sub-assembly consisted of two beams in one direction, and one beam in the orthogonal direction. All three beams in the sub-assembly were one-half the bay length and the column extended to one-half the inter-storey height of the prototype building in both directions; this assumes that the point 
of inflexion occurs at the midpoints of the beams and column. An external damping system was used to provide additional energy dissipation.

The column was $3.2 \mathrm{~m}$ high and had a square section of $700 \mathrm{~mm}$ x $700 \mathrm{~mm}$ dimensions. Axial load due to self weight of the above floors was estimated as $2000 \mathrm{kN}$ and simulated through prestressing consisting of four $32 \mathrm{~mm}$ diameter high strength threaded rod bars $\left(\right.$ Macalloy $\left.{ }^{\mathrm{TM}}\right)$. Three $20 \mathrm{~mm}$ thick mild steel plates were cast at column faces where precast beams were jointed. The minimum reinforcement ratio $\left(\rho_{l}=0.008\right)$ was provided using 12 HD 20 threaded bars (Reidbars ${ }^{\mathrm{TM}}$ ). This low reinforcement ratio prevented over-crowding of reinforcing steel in the joint region; thereby reducing construction difficulties. Reinforcing details of the column are shown in Figure 1a. In Figure 1a, the specimen design is symmetrical about the vertical centreline of the column. However, for clarity some of the details of Figure 1a have been omitted (i.e. no post-tensioning or mild steel reinforcing shown in the left beam, and the dissipator on the right beam has also been omitted). To transfer shear forces through the joint, five double HR12 hoop sets at $100 \mathrm{~mm}$ centre-to-centre spacing were placed in the joint. At both ends of the column, four sets of $100 \mathrm{~mm}$ centre-to-centre spaced stirrups were used to disperse the post-tensioning force. By assuming that the concentrated force which occurs in the column, due to rocking of the beam on an edge (2D planar excitation) or a corner (3D excitation), spreads at an angle of 45 degrees through the plate, the thickness of the steel plate was calculated to be $20 \mathrm{~mm}$.

The two seismic beams in the same longitudinal direction formed part of the seismic resisting frame, while the gravity beam in the orthogonal (transverse) direction represented part of the frame used to seat a one-way precast concrete hollowcore floor system. Layouts of reinforcing bars in the precast beams are shown in detail in Figures 1a, 1b and 1c. All beams were $4 \mathrm{~m}$ long and had cross-sectional dimensions of $400 \mathrm{~mm} \times 560 \mathrm{~mm}$. The beams were prestressed with two $26.5 \mathrm{~mm}$ high-strength $\left(\mathrm{f}_{\mathrm{y}}=1000 \mathrm{MPa}, \varepsilon_{\mathrm{y}}=0.052, \varepsilon_{\mathrm{u}}=0.06\right)$ high-alloy 
threaded bars (Dywidag ${ }^{\mathrm{TM}}$ ). A straight tendon profile connected with a short diagonal fuse rod near the column was designed for the seismic beams, while a draped tendon profile was adopted for the gravity beam to represent balanced dead load. To protect beam ends from large concentrated forces occurring during rocking behaviour, two $100 \mathrm{~mm} \times 100 \mathrm{~mm} \times 12$ $\mathrm{mm}$ steel angles were cast at the top and bottom edges of the beams at the connection.

A cracked elastic design was used to detail longitudinal reinforcement in the precast beam segments. In this design approach, sufficient reinforcing bars are provided to ensure that yield of longitudinal reinforcement is prevented and concrete compressive stresses remain below $0.7 f^{\prime} c$. This ensures that the precast elements remain essentially elastic even when the connection reaches over-strength. Shear design of the precast elements was done using the New Zealand standard NZS 3101 [15]. With a total axial load of $398 \mathrm{kN}$ (provided by posttensioning the two threaded bars to $f_{i}=0.5 f_{y}$ ), the required transverse reinforcement was less than for a conventional system. Hence in the central portion of the beams, only minimal transverse steel was used, thus the maximum allowable stirrup spacing of $250 \mathrm{~mm}$ centre-tocentre was adopted. Closely spaced (100 mm centre-to-centre) stirrups were placed at beam ends to help transfer large rocking and post-tensioning forces.

One important aspect in ensuring that jointed precast systems are adopted in the construction industry is ease of erection, of which a major component is the method of posttensioning. For this design the high strength threaded bars were placed in PVC ducts off-site with threaded couplers attached to the thread bar at the end near the connection as shown in Figures 1a and 1c. Fuse bolt bars are then used to connect to the coupler and run through the column and fastened on the other side. The name fuse is used because these short and replaceable bolt bars are milled down to $75 \%$ of their original area; therefore they act as the weakest link and completely rule out the possibility of yielding of the prestressing tendon. Once the beams are correctly in place, bolt bars are tensioned separately to give added 
redundancy to the structure in the unlikely case of tendon or anchorage failure.

Because a straight tendon profile was adopted for the seismic beams a bent coupler at an angle of 20 degrees was required to enable the fuse bolt bars to pass diagonally through the column, and be fastened above the opposing seismic beam. For the gravity beam however, as there was no beam on the opposite side of the column, there was no need to have a coupler with a large inclination. A straight coupler was therefore used for the bottom tendon, and a coupler at an angle of 10 degrees for the top draped tendon. Four tapered shear keys (Figure 1c) were used at beam-column interfaces to carry shear forces, and to provide torsional restraint. The steel shear keys were fitted in steel slots on the angles of the beams.

In conventional monolithic structures, seismic energy imparted to the structure during earthquake excitation is dissipated through the development of plastic hinges at critical locations which cause damage requiring post-earthquake repair. In jointed precast systems designed in accordance with DAD principles the drift demand is accommodated via gap opening at the rocking connections, and the energy dissipation due to plastic hinge formation does not occur. It has been previously claimed [2] that jointed precast systems with insufficient energy dissipation capacity are subjected to higher displacement demands than equivalent ductile monolithic structures. It is therefore necessary to have a supplemental damping system to provide this required energy dissipation, while maintaining the DAD philosophy by limiting damage to replaceable components. Tension-compression dissipators in the form of machined mild steel rods were used for these experiments. These dissipators were connected across the beam-column interface by screwing to the column at one side and to steel plates cast into the beams at the other side (Figures 1a and 1c). Further details of the specimen design are given in [11].

\section{Loading Protocol}

In order to perform MSPA, the earthquake records to be used must be pre-identified. 
Dhakal et al. [13] have proposed a methodology based on incremental dynamic analysis (IDA), in which IDA is conducted using several earthquake records and the IDA results are probabilistically processed to identify records that give medium and high confidence at desired levels of seismic intensity. IDA [16] involves conducting non-linear dynamic analyses of a computational model of a structure subjected to a suite of earthquake ground motion records scaled to different intensity measures (IM). For each analysis, an engineering demand parameter (EDP) is monitored, thus producing an IDA curve (i.e. a plot of IM vs. EDP) for each earthquake record.

For this study, two suites of 10 bi-directional earthquake records were used; the details of the records in these two suites are presented in $[17,18]$. One suite consists of ground motion records from near-source earthquakes (i.e. near-field, high intensity records) and the other suite includes records from medium-source earthquakes (i.e. medium-field, medium intensity records). The purpose of using these two suites of earthquake records was to compare the response of different source mechanisms. To allow the records to be used for both bi-directional and uni-directional analyses, the fault-normal and fault-parallel components of the unprocessed records were combined, rotated 45 degrees and resolved into two directions, thus removing characteristic fault-normal, fault-parallel behaviour of the original records.

In order to perform IDA, an analytical model of the beam-column joint sub-assembly was conceptualised using Ruaumoko3D [19]. The beams and columns were represented using frame elements with an elastic hysteresis, which is inline with the expectation of DAD philosophy that all components/members (except for the rocking connections) behave elastically. The inelastic and nonlinear behaviour of the rocking joint was described using two springs of zero length in parallel (Figure 2a). The first spring represented the behaviour of the unbonded post-tensioning tendon and had a tri-linear elastic hysteresis to represent the 
pre-rocking, gap-opening, and tendon-yielding phases, respectively. The second spring was elastoplastic and represented the supplemental energy dissipation system. The assumption of a tri-linear elastic hysteresis for the effect of the post-tensioning, physically means that following yielding (i.e. the third branch of the hysteresis) no energy is dissipated. This is obviously incorrect, (in reality from the point of load reversal the unloading slope would be the same as the second slope until it intersected the elastic slope) however based on the specimen design, yielding of the threaded bars would not occur until $4.6 \%$ drift, and hence this modelling assumption really only effects the collapse capacity of the specimen and not the drift limits imposed in the experimental program.

The parameters of the springs representing the rocking joints were calibrated based on the results of preliminary quasi-static tests [11]. These preliminary tests were up to drifts of 2\%, which was sufficient to characterise: (i) the initial stiffness; (ii) drift at gap opening; (iii) post gapping stiffness; and (iv) energy dissipation. The drift at which tendon yield occurred was based on design calculations given elsewhere [11]. Although it has been shown in some cases that the Monolithic Beam Analogy [20] can approximate these points relatively well, the preliminary tests were conducted for further verification, which was enabled due to the 'damage-free' properties of the specimen. A typical comparison between the experimental and calibrated analytical results is shown in Figure $2 \mathrm{~b}$ for unidirectional cyclic test up to $2 \%$ drift.

A computational model of the 10-storey prototype building was developed. IDA was conducted using the two suites of ground motion records. The IM selected was the 5\% damped elastic spectral acceleration at the fundamental time period of the model, which was 1.6 seconds, i.e. $I M=S_{a}\left(T_{1}, 5 \%\right)$. Assuming that the fundamental period of the structure $T_{1}$ lies in the constant velocity range of the design acceleration spectra, the spectral acceleration can be related to the PGA by $S_{a}=P G A / T_{1}$ (alternatively, if available the $S_{a}$ values for certain 
exceedance frequencies could be obtained directly from a seismic hazard curve of $S_{a}$ ). Hence, the spectral accelerations for the design basis earthquake (DBE) with $10 \%$ probability of occurrence in 50 years $(0.4 \mathrm{~g}$ PGA for the design location of Wellington, NZ) and maximum considered earthquake (MCE) with $2 \%$ probability of occurrence in 50 years $(0.72 \mathrm{~g}$ PGA for Wellington, NZ) are $0.25 \mathrm{~g}$ and $0.45 \mathrm{~g}$, respectively. These spectral acceleration values are used as the IM representing DBE and MCE (see Figure 2c), which have direct relevance in performance based seismic design and hence in MSPA, as will be explained later.

Each orthogonal pair of earthquake records were compared and the larger component was applied along the seismic frame direction, with the smaller component applied in the gravity frame direction. This orientation was chosen based on a separate investigation using 5 uni-directional earthquakes applied in: (1) the seismic frame direction; (2) the gravity frame direction; and (3) at 45 degrees from the two principal directions. The response interstorey drifts were the highest when the earthquakes were applied in the seismic frame direction. The use of this simplified approach was thought to be acceptable as the focus of this study was not to investigate the effect of directivity in 3-D modelling.

The EDP is an indicator of the level of demand that the structure is subjected to due to the earthquake ground motion. Although several options are possible, the EDP used in this study was the maximum interstorey drift occurring in either of the two principal frame directions, as it relates well to joint rotations and both local and global collapse. Another typical candidate EDP is cumulative plastic rotation.

These analyses resulted in a matrix of data points on an EDP (i.e. maximum inter-storey drift $\left.\theta_{\max }\right)$ versus IM $\left(S_{a}\right)$ plot, which were connected to obtain the IDA curve for all earthquake records in the suites. The resulting IDA curves for the suite of near-source earthquakes are shown in Figure 2c. From the generated IDA curves the structural behaviour was assessed at three different levels of performance. The first performance criterion is that 
at the DBE intensity level, there should be a high probability (assumed 90\%) that the structure will suffer no more than minor damage, to ensure immediate occupancy. The second criterion is that at the MCE intensity level there should be a moderate probability (assumed $50 \%$ ) that structural damage will be repairable. Finally, the third criterion is that at the MCE intensity level there should be a high probability (assumed 90\%) that global collapse of the structure will not occur, to ensure life-safety. Hence, from each of the two suites, 3 records were selected that are closest to the $90^{\text {th }}$ percentile response at the DBE intensity level (i.e. $S_{a}=$ $0.25 \mathrm{~g}$ ) and the $50^{\text {th }}$ and $90^{\text {th }}$ percentile responses at the MCE intensity level (i.e. $S_{a}=0.45 \mathrm{~g}$ ). The near-source ground motion records identified to represent these three seismic hazard levels are illustrated in Figure 2c, and the seismological details of the three earthquakes for the two suites are listed in Table 1. These earthquakes were used in MSPA of the building through QED testing of the specimen (although it is noted that either pseudo-dynamic or shake table tests could be used in the MSPA for the selected ground motion records).

For the QED tests, displacement-history at the loading point (top of the column) was required. Nonlinear dynamic time-history analyses were carried out on the 10-storey building model using these critical earthquakes, and the displacement response of the nodes at the centre of the columns on the second and the first floor were recorded. As these nodes represented the top and bottom of the column in the sub-assembly to be tested, the difference between the displacements of these two nodes gave the inter-storey displacement for the beam-column sub-assembly. Thus, bi-directional inter-story drift histories were deduced and applied to the top of the column through two hydraulic actuators.

\section{Test Setup and Instrumentation}

Figures $3 \mathrm{a}, 3 \mathrm{~b}$, and $3 \mathrm{c}$ present plan view and elevation views of the experimental setup. Loading was performed by three hydraulic actuators (Ram A, B, C). Two large actuators, one along the East-West direction (Ram A), and the other along the North-South direction (Ram 
B) were installed between reaction frames and the top of the column. A third actuator (Ram

C) was installed along the East-West direction at the end of the gravity beam to keep the specimen movement in-plane when performing unidirectional tests, and also to measure the amount of torsion in the specimen during bidirectional testing (if any). Ram C was synchronised with Ram A such that it moved only half of the movement of Ram A (since the height of Ram C from the bottom of the specimen is half that of Ram A). Gravity load was applied at the top of the gravity beam through a $300 \mathrm{kN}$ capacity hydraulic jack to simulate loads applied from the one-way flooring system. A 1.5 meter long, $200 \mathrm{~mm}$ deep Laminated Veneer Lumber (LVL) block was used to spread the gravity loads uniformly along the beam, and a pressure release valve was used to maintain a constant gravity load during testing.

Altogether, twelve load cells were used to measure the forces in hydraulic rams and prestressing forces in the threaded bars. Three $50 \mathrm{~mm}$ stroke linear potentiometers were installed along each face of the three rocking connections to monitor connection opening. Two $100 \mathrm{~mm}$ stroke linear potentiometers were installed underneath precast beams near rocking connections to capture vertical movements. Eight string potentiometers were placed around the specimen to capture any out-of-plane movement. Strain gauges with $5 \mathrm{~mm}$ gauge length were used to monitor longitudinal strains in the fuse bolt-bars. Two gauges were attached (at one quarter of the fuse length) on the surface of each supplemental energy dissipator.

The data acquisition system used in this experiment was composed of two parallel subsystems: (1) the controller subsystem; and (2) the data logging subsystem. In the data logging subsystem, all measurements (i.e. displacements, rotations and strains) were recorded through data loggers. A controller interface programme read the displacement-time history from the input file and activated the hydraulic rams. At the end of each time interval, the specimen movement was recorded through a controller box and compared to the target 
movement read from the input file. When the measured movement was equal to the target movement (within negligible limits) the programme initiated the next target movement from the input file. A non-linear controller was used to ensure that overshoot/undershoot was not significant.

Further details of the experimental setup are given in [11].

\section{EXPERIMENTAL RESULTS}

As mentioned previously, three earthquakes records were identified from the two suites for conducting the MSPA. Therefore, six tests were performed to verify the three performance-based seismic design requirements. The final column of Table 1 shows the peak drift responses obtained from IDA, which were applied in the six tests conducted. According to the concept of MSPA, the six records were applied to the sub-assembly in the order of increasing drift demand. Here, experimental results are presented in detail only for the larger of the two responses at each performance assessment level.

\section{Response to the $90^{\text {th }}$ Percentile DBE}

The near source ground motion causing the $90^{\text {th }}$ percentile response at DBE (with a return period 475 years) was EQ40 which induced a maximum drift of $2.1 \%$. Figures $4 d$ and $4 \mathrm{f}$ shows the input drifts applied to the sub-assembly, while Figure $4 \mathrm{c}$ shows the drift orbit of the top of the column. Figures $4 \mathrm{e}$ and $4 \mathrm{~g}$ show gap opening on the east and west seismic beams respectively at a drift of $2.13 \%$. Note that as previously mentioned, preliminary quasistatic tests were conducted on these specimens [11] whose results were used to calibrate the analytical models. Moreover, the displacement time-history corresponding to EQ7 $\left(90^{\text {th }}\right.$ percentile DBE medium source record) had also been applied to the sub-assembly prior to the QED test for EQ40. During these tests, some cracks had emerged in the beam near the rocking interface. Consequently, some cracks in the top-half depth and minor spalling in the 
inside of the bottom steel angle can be seen in Figure 4g.

Figures $4 \mathrm{e}$ and $4 \mathrm{~g}$ show a gap opening of approximately $5 \mathrm{~mm}$ at the edge of the beam, however at the mid-depth of the beam there is no gap opening, indicating that the expectation of rigid body movement at the rocking interface was not fully achieved. Due to the nature of near source records, the input drifts are comprised primarily of one peak occurring simultaneously in both orthogonal directions. The peak drifts on this cycle were $1.95 \%$ and $0.64 \%$ in EW and NS directions, respectively. The corresponding lateral forces for the EW and NS directions were $118.9 \mathrm{kN}$ and $38.1 \mathrm{kN}$, respectively. On the maximum drift cycle, the supplemental energy dissipators on the seismic beams yielded partially, however no noticeable buckling occurred during unloading. The partial yielding of the dissipators is also indicated by the moderate energy dissipation occurring during the peak drift cycle (Figure 4b). Overall, the specimen performed satisfactorily, and as there were no visual signs of damage requiring repair then it can be concluded that no repair of the structural system would be required to restore functionality after this test.

\section{Response to the $50^{\text {th }}$ Percentile MCE}

The near source ground motion causing the $50^{\text {th }}$ percentile response at MCE (with a return period 2475 years) was EQ30 which induced a maximum drift of $2.8 \%$. Figures $5 \mathrm{f}$ and 5h show the input drifts in the two directions, while Figure 5e shows the drift orbit of the top of the column. The peak drifts were $2.8 \%$ in the EW direction and $1.77 \%$ in the NS direction. The corresponding lateral forces in EW and NS directions were $149.4 \mathrm{kN}$ and $77.5 \mathrm{kN}$, respectively. As can be seen in Figures $5 \mathrm{~b}$ and $5 \mathrm{~d}$, the drifts are not exactly zero when the lateral load was fully unloaded. It was similar during the quasi-static tests conducted for the verification of the computational model (Figure $2 \mathrm{~b}$ ) and during the previous QED tests with EQ7 and EQ40 (Figures 4a and 4b). This apparent lack of re-centering capability in the lateral load vs. drift plot was due to the large friction forces that occurred between the bolt bar 
(used for connecting the straight tendon and the inclined fuse bar to form the post-tensioning system) and the encasing PVC duct, which are accentuated with the bent coupler profile. The bent coupler profile also resulted in the occurrence of shuddering noises when friction between the duct and the bolt bar was overcome and slip occurred.

The lateral load vs. drift hysteretic response in the NS direction (Figure 5b) shows good energy dissipation during the peak drift cycles. Figure 5i shows the gravity-beam to column connection at NS drift of $1.5 \%$, where the beam undergoes 'partial rocking' (when the beam at the beam-column interface does not rock (almost) rigidly on the column face, due to the insufficient stiffness of the steel angles). Upon re-centering of the gravity beam from the maximum drift cycles in either direction, buckling of supplemental energy dissipators occurred (Figure 5g). The lateral load vs. drift response in the EW direction (Figure 5d) shows very good energy dissipation during the peak drift cycles, which occurred at 18 and 21 seconds in the positive and negative directions, respectively. However, in the aftermath of these large drift cycles, energy dissipation at subsequent cycles of lower drift is noticeably smaller. This is thought to be due mainly to the buckling of the external energy dissipators.

Minor strength degradation is apparent during the two large cycles in the negative EW drift direction (Figure 5d). As the damage in the specimen at this stage was not significant, it was deduced that damage was not contributing much to the strength degradation. The cause of this is believed to be the reduction in efficiency of dissipators due to buckling, which occurred during unloading (i.e. re-centering) from the previous large drift cycle in the positive EW direction. Figures 5a and 5c show the lower steel angle on the west-side seismic beam viewed from the north and south, respectively. Minor crushing and spalling from the previous tests caused a reduction in load carrying capacity of concrete around these affected regions, and at the peak drift of $2.84 \%$ some spalled concrete fell off the beam. The lack of apparent recentering mentioned previously appeared less significant as the absolute value of the drift 
increased. It suggests that friction forces between the bolt bar and the PVC duct is of little concern for high drift responses, where residual drifts are in the order of $0.2 \%(6 \mathrm{~mm})$. After the test, it was apparent that the external energy dissipators needed to be replaced. It is expected that this could be managed without causing any significant disruption to the building occupants, since the dissipators are external (as opposed to internal) of the structural system. However, in accordance with the MSPA methodology (that one specimen is required), the dissipators were not replaced before the 90\% MCE experiment. The 'carry-over damage' effects of this are noted in the discussion section.

\section{Response to the $90^{\text {th }}$ Percentile MCE}

The medium source ground motion generating the $90 \%$ percentile response at the MCE intensity level was EQ11 which induced a maximum drift of $4.7 \%$. Figure $6 \mathrm{~g}$ shows an elevation of the specimen at the maximum drift. The QED test results for EQ11 are shown in various forms in Figure 6. Figures $6 \mathrm{f}$ and $6 \mathrm{~h}$ show the input drifts in the two directions, while Figure 6e shows the drift orbit of the top of the column. Peak drifts were $4.7 \%$ in the EW direction and $3.5 \%$ in the NS direction. The corresponding lateral forces in EW and NS directions were $171.5 \mathrm{kN}$ and $77.8 \mathrm{kN}$, respectively. Note that unlike the five previous tests which had drift demands in the NS (gravity-frame) direction less than $40 \%$ of that in the EW (seismic-frame direction), the NS drift demand in this test was more than $70 \%$ of the EW drift demand.

The lateral load vs. drift response in the NS direction is shown in Figure 6b, which shows good energy dissipation during the peak drift cycles in both positive and negative directions. No significant damage occurred in the gravity beam other than very minor spalling at corners of the connection and a few hairline flexural cracks. The EW lateral load vs. drift hysteresis is also shown in Figure 6d. It can be seen that good energy dissipation was provided by the external energy dissipation system. The peak drift of $4.68 \%$ in the EW 
direction caused additional damage to the beam in the form of concrete spalling/crushing at rocking edges as shown in Figure 6c. Large drifts in the EW direction also led to slip in the connection between the energy dissipators and column, which occurred after un-loading from the maximum positive drift of $4.25 \%$. It can be seen that after the maximum drift cycles in both NS and EW directions, energy dissipation for subsequent smaller drift cycles is relatively small (Figure 6d). This occurred because of the buckling of dissipators (Figure 6i) upon recentering from the maximum drift cycles.

As can be observed from the hysteresis loop corresponding to the maximum EW drift of $4.7 \%$ in Figure $6 \mathrm{~d}$, a flat plateau exists near the peak and also a significantly higher energy dissipation is apparent in the hysteresis loop. This is because of yielding of all four fuse bars in the EW beams. Despite this yielding, the sub-assembly's re-centering capability did not deteriorate; it had a residual drift of only $0.2 \%(6 \mathrm{~mm})$. However, inelastic strain caused by yielding of the tendons did reduce post-tensioning forces, and as a result the bi-linear stiffness of the sub-assembly reduced during subsequent cycles. The test indicated that the specimen needed to be repaired and the post-tensioning tendons may need to be tightened to overcome the loss of prestress level due to yielding.

\section{DISCUSSION: EXPECTED PERFORMANCE OF THE BUILDING WITH RESPECT TO THE SEISMIC DESIGN CRITERIA}

Earthquake records representing different levels of seismic hazard were applied in sequence to the same specimen to assess the performance of the specimen with respect to different levels of seismic design requirements. The test results can now be interpreted inline with the principles of MSPA to verify if the different seismic performance criteria were met. In other words, seismic performance of the building at the three different levels of seismic demand can be extrapolated based on these test results. 
The first level of seismic performance is to review the response of the structure to the designed level of earthquake: i.e. DBE with an intensity of $0.4 \mathrm{~g}$ PGA $\left(S_{a}=0.25 \mathrm{~g}\right)$. At this level of seismic hazard, one needs to have high confidence that no significant damage needing major repair will occur (so that the structure keeps functioning without any disruption after the earthquake). In fact, there exists, in many cases, one more level of performance requirement prior to this; that is the structure should have no damage at all during frequent earthquakes. These frequent earthquakes have intensities significantly less than that of DBE, and this preliminary seismic performance requirement has not been considered in this study. To ensure that the structure will suffer no more than slight/minor damage due to the DBE, the structure needs to be tested against an earthquake likely to induce the largest response and cause severest damage at the DBE intensity level than any other earthquakes scaled to the same intensity level. EQ40 was selected for this purpose because it generated the $90^{\text {th }}$ percentile response at DBE level among the 40 earthquakes in the suite. During the $90^{\text {th }}$ percentile DBE test, no new cracks were observed in the members, although minor cracks formed during the previous quasi-static and previous QED tests were present. Although the external dissipators yielded partially, buckling was not evident and replacement of the dissipators was not required. The sub-assembly therefore satisfied the first requirement of the performance based seismic design.

The second and third levels of seismic performance assessment refer to structural response at rare earthquakes; i.e. MCE with an intensity of PGA $=0.72 \mathrm{~g}\left(S_{a}=0.45 \mathrm{~g}\right)$ for the location of Wellington, NZ used in this study. The second performance requirement indicates that there must be moderate confidence that the induced damage will be repairable due to an earthquake of MCE intensity level. This requirement ensures that a fair possibility exists that the structure will not need to be dismantled and can be repaired to regain its functionality even after rare and large earthquakes. To prove that there exists a moderate probability of the 
structure suffering only repairable and moderate damage after the maximum considered level of seismic hazard, the structure needs to be tested against an earthquake giving moderate confidence (assumed 50\% in this study) of representing the MCE intensity level. EQ30 generated the $50^{\text {th }}$ percentile response at MCE level among the 40 earthquakes selected, and generated a peak drift of 2.84\%. QED tests using this record (i.e. EQ30) caused buckling of the external energy dissipators upon re-centering, which resulted in reduced energy dissipation during the subsequent drift cycles. No strength degradation of beams or column were observed, however buckling of the energy dissipators caused minor global strength degradation. Some spalled concrete fell off the specimen at the peak drift. In order to restore the specimen following this $50^{\text {th }}$ percentile MCE test, only minor patching of spalled concrete around steel angles, and replacement of external dissipators would be required. Therefore, it can be concluded that the structural performance was adequate to satisfy the second performance requirement.

The third and final seismic performance requirement demands that there must be high confidence that the structure will not collapse in a rare earthquake; i.e. represented by MCE with a $2 \%$ probability in 50 years. This requirement is to ensure life-safety of people even in a rare and large earthquake. To ensure that the structure will suffer severe damage (at most) and will not collapse due to the maximum considered level of seismic hazard, the structure needs to be tested against an earthquake giving high confidence (assumed $90 \%$ in this study) of representing the MCE intensity level. EQ11 generated the $90^{\text {th }}$ percentile response at the MCE intensity level and had a peak drift of 4.69\%. QED test using EQ11 caused spalling of concrete in the seismic beams at the beam-column connections, severe buckling of the supplemental energy dissipators, yielding of post-tensioning tendons, and minor flexural cracks in the beams. Note that these damages were also contributed by quasi-static tests and the five QED tests conducted prior to this test. No significant strength degradation however 
was observed in the lateral load vs. drift curve obtained from this test. Yielding of the posttensioning threaded bars in the experiment did not affect the re-centering capability of the sub-assembly. Repair work required would be replacement of external dissipators, patching of spalled concrete and re-stressing of the yielded threaded bars. Most importantly, the specimen did not collapse (the threaded bars are ductile, so minor yeilding (e.g. $\varepsilon_{\max }=0.01$ ) is acceptable without significant risk of collapse). It can therefore be concluded that the subassembly easily satisfied the third and final performance based design criteria.

Thus, the proposed MSPA methodology can be used to verify multiple seismic performance requirements by testing a single specimen. One apparent limitation of this procedure is that the damage from earlier tests are carried over to the latter tests; thereby overestimating the damage during the latter tests. Note that the damage is expected to be little (or non-existent) in the first test (verifying the immediate occupancy criteria) and the carryover effect to the second test is hence negligible. Nevertheless, slight to moderate damage (within repairable range) may be expected in the second test which will be carried over to the final test. This damage carryover results in a stricter measure of the final seismic design criteria (i.e. life-safety), which is desirable given the harsh consequence if this criterion is not met.

It is also noted that existing shake-table of pseudo dynamic test procedures (which use the same ground motion record scaled to various levels of intensity) effectively relate to having only one line on the IDA plot given in Figure 2c. Hence by conducting these existing test procedures, the experimenter has no concept of the confidence in the performance of the structure at various levels of ground motion intensity. This therefore illustrates the need for the MSPA in experimental testing of structures subjected to earthquake loadings. 


\section{DISCUSSION: PERFORMANCE OF DAMAGE AVOIDANCE DESIGN AND DETAILING}

Overall, the specimen performed well in all six QED tests. Although designed for the DBE, the specimen could sustain MCE without severe damage and collapse. The authors believe that a contemporary ductile specimen designed for DBE would have been significantly damaged in a 90\% MCE event, based on observed structural damage during past earthquakes, and also since the design philosophy is based on incurring significant localised damage in plastic hinge regions. Despite its superior performance in comparison with ductile frames, the experimental results did not fully satisfy the essence of the DAD philosophy; that is damage avoidance. No doubt the damage was restricted and controlled, but not avoided. Some discussion on the performance of different components/details follows.

Shear keys: - Figure 6a shows the exposed shear keys at the maximum drift of $4.8 \%$ during the final test. As no problems occurred in the shear keys during all six tests, the performance of the shear keys at high drift levels was verified. Large axial rotation of the gravity beam was observed during the maximum drift in the seismic frame direction, indicating the adequacy of shear keys in providing torsional restraint.

Rocking interface details: - The main requirement for details in the rocking interface is to transfer the concentrated rocking force to the concrete behind the interface without causing significant stress concentrations in the concrete leading to damage. Clearly, the steel angle implemented in the specimen could not fully satisfy this criterion. Experimental results show partial rocking due to stress concentrations at rocking edges. Based on Saint Venant's principle this disturbed region will continue to approximately one beam depth into the beam length. The observed softening effect is primarily because of insufficient stiffness provided by steel angles, which resulted in minor spalling of concrete behind the angles. Further attention needs to be directed to beam-column connection details to ensure that beams are 
forced to rock about the toe of the beam, giving an increased initial stiffness and high energy dissipation at low drift cycles.

Coupler and bolt bar details: - In general, the prestressing tendons worked well. Coupling fuse bolt bars with reduced cross-section near the connection ensured that yielding occurred in the fuse, if at all. This renders post-earthquake repair (i.e. replacing the fuse bars) possible and easier. The bent profile of the coupler resulted in shuddering noises during the tests when slip occurred between the encasing PVC duct and the bolt bar. Nevertheless, its structural performance was adequate, allowing almost full re-centering after removal of the lateral loads.

Supplemental energy dissipator: - The performance of the damping system was verified as the hysteretic curves showed large energy dissipation. Nevertheless, the dissipators buckled while re-centering after large drift cycles. More robust energy dissipating devices need to be developed to provide high damping for a broad range of loading regimes, while keeping with the DAD philosophy of being easy to replace if damaged.

\section{CONCLUSIONS}

The following conclusions can be drawn from this study:

1. A Multi-level Seismic Performance Assessment (MSPA) methodology has been established, in which earthquake records representing different levels of seismic hazard are pre-identified and applied in sequence of increasing demand to a single specimen to verify multiple seismic performance requirements. The MSPA methodology apparently has two advantages over the regular quasi-static displacement-regulated tests: it gives more accurate indication of likely performance of the structure during earthquakes, and it saves significant amount of resources that would have otherwise been needed to test multiple specimens to verify the different seismic performance criteria. 
2. The MSPA methodology has been applied to a 10-storey moment resisting RC frame building designed according to Damage Avoidance Design (DAD) philosophy. QuasiEarthquake Displacement (QED) tests were conducted on a 3D beam-column joint subassembly from a critical part of the building frame, which was sequentially subjected to the analytically predicted displacement histories due to the three pre-identified earthquake records.

3. Based on the experimental results presented herein:

a. One can be some $90 \%$ confident that the 10 -storey DAD building (the subassembly is a critical part of this building) will survive a Design Basis Earthquake (DBE) without needing a major repair and without causing any downtime.

b. Similarly, one can be at least $50 \%$ confident that the DAD building can survive a Maximum Considered Earthquake (MCE) without needing to dismantle it. After some downtime needed to repair the incurred damage, the building could be reused after an MCE.

c. One can also be at least $90 \%$ confident that the DAD building will not collapse in an MCE, thereby ensuring the most important requirement of life safety.

4. Design and detailing procedures for beams and columns were shown to be adequate with the column sustaining no damage throughout all experiments, and beams suffering only minor flexural cracks. In general, the survival of the sub-assembly up to drifts of $4.7 \%$ with negligible strength degradation and residual drift verified superior seismic performance of the DAD philosophy. Nevertheless, design details (specially the interface details and the energy dissipators) need to be improved if the damage avoidance requirement is to be strictly/literally satisfied. 


\section{REFERENCES}

1. Housner GW. The behaviour of inverted pendulum structure during earthquake. Bulletin of the Seismological Society of America 1963, 53(2):403-417.

2. Cheok GS, Lew HS. Performance of precast concrete beam to column connections subject to cyclic loading. PCI journal 1991, 36(3):56-67.

3. Cheok GS, Lew HS. Model precast concrete beam-to-column connections subject to cyclic loading. PCI Journal 1993, 38(4):80-92.

4. Priestley MJN, Tao JRT. Seismic response of precast prestressed concrete frames with partially debonded tendons. PCI Journal 1993, 38(1):58-69.

5. Stone WC, Cheok GS, Stanton JF. Performance of hybrid moment-resisting precast beam-column concrete connection subjected to cyclic loading. ACI Journal 1995, 91(2).

6. Priestley MJN, Sritharan S, Conley JR, Pampanin S. Preliminary results and conclusions from the PRESSS five-storey precast concrete test building. PCI Journal 1999, 44(6):43-67.

7. Mander JB, Cheng CT. Seismic resistance of bridge piers based on damage avoidance design. Technical Report NCEER-97-0014 (National Centre of Earthquake Engineering Research) 1997, Department of Civil, Structural and Environmental Engineering, State University of New York at Buffalo, New York, USA.

8. Solberg K, Mashiko N, Dhakal RP, Mander JB. Performance of a damage-protected highway bridge pier subjected to bi-directional earthquake attack. Proceedings of the $19^{\text {th }}$ Australasian Conference on the Mechanics of Structures and Materials (ACMSM19) 2006, Christchurch, NZ, pp. 437-442.

9. Hamid NBA, Mander JB. Experimental study on bi-lateral seismic performance of precast hollow core wall using shaking table. Proceedings of the $10^{\text {th }}$ East Asia and Pacific Conference on Structural Engineering and Construction (EASEC10) 2006, Bangkok, Thailand, Vol. 7.

10. Holden TJ, Restrepo JI, Mander JB. Seismic performance of precast reinforced and prestressed concrete walls. Journal of Structural Engineering 2003, 129(3): 286-296

11. Li L. Further experiments on damage avoidance design of beam-to-column joints. $M E$ Thesis 2006, Department of Civil Engineering, University of Canterbury, Christchurch, New Zealand. http://library.canterbury.ac.nz/etd/adt-NZCU20060828.153252

12. Arnold DM. Development and experimental testing of a seismic damage-avoidance designed beam to column connection utilising draped unbonded post-tensioning. $M E$ Thesis 2004, Department of Civil Engineering, University of Canterbury, Christchurch, New Zealand.

13. Dhakal RP, Mander JB, Mashiko N. Identification of critical earthquakes for seismic 
performance assessment of structures. Earthquake Engineering and Structural Dynamics 2006, 35(8):989-1008.

14. Bull D, Brunsdon D. Examples of concrete structural design to New Zealand Standard NZS3101. Cement and Concrete Association of New Zealand (CCANZ) 1998, New Zealand.

15. Standards New Zealand. NZS 3101: Part 1: 1995: Concrete Structures Standard. Standards New Zealand 1995, Wellington.

16. Vamvatsikos D, Cornell CA. Incremental dynamic analysis. Earthquake Engineering and Structural Dynamics 2002, 31:491-514.

17. SAC Steel Project. Los Angeles Earthquake Ground Motions with 10\% Probability of Exceedance in 50 years. SAC Steel Project. Earthquake Ground Motions for the Analysis of Steel Structures http://nisee.berkeley.edu/data/strong_motion/sacsteel/ motions/la10in50yr.html.

18. SAC Steel Project. Los Angeles Earthquake Ground Motions with 2\% Probability of Exceedance in 50 years. SAC Steel Project. Earthquake Ground Motions for the Analysis of Steel Structures. http://nisee.berkeley.edu/data/strong_motion/sacsteel/ motions/la2in50yr.html.

19. Carr AJ. Ruaumoko3D: Inelastic Dynamic Computer Program. Computer Program Library 2004, Department of Civil Engineering, University of Canterbury, Christchurch, New Zealand.

20. Pampanin S, Priestley NJ, and Sritharan S. Analytical modelling of the Seismic Behaviour of Precast Concrete Frames Designed with Ductile Connections. Journal of Earthquake Engineering 2001, 5(3):329-367. 
Table 1: Earthquake Records selected from IDA

\begin{tabular}{|c|c|c|c|c|c|c|c|c|c|c|c|}
\hline ID & Event & Year & Station & $\mathrm{M}^{* 1}$ & $\begin{array}{l}\mathrm{R}^{* 2} \\
(\mathrm{~km})\end{array}$ & Component & $\begin{array}{c}\mathrm{S}_{\mathrm{A}}^{* 3} \\
(\mathrm{~g})\end{array}$ & Source & $\begin{array}{c}\text { Hazard } \\
\text { level }\end{array}$ & $\begin{array}{l}\mathrm{S}_{\mathrm{A}}{ }^{* 4} \\
(\mathrm{~g})\end{array}$ & $\begin{array}{c}\text { Peak } \\
\text { Drift } \\
(\%)\end{array}$ \\
\hline \multirow{2}{*}{ EQ7 } & \multirow{2}{*}{ Landers } & \multirow{2}{*}{1992} & \multirow{2}{*}{ Barstow } & \multirow{2}{*}{7.3} & \multirow{2}{*}{36} & EW & 0.18 & \multirow{2}{*}{ medium } & $90 \%$ & 0.25 & \multirow{2}{*}{1.2} \\
\hline & & & & & & NS & 0.093 & & DBE & 0.13 & \\
\hline \multirow{2}{*}{ EQ1 } & \multirow{2}{*}{$\begin{array}{c}\text { Imperial } \\
\text { Valley }\end{array}$} & \multirow{2}{*}{1940} & \multirow{2}{*}{ El Centro } & \multirow{2}{*}{6.9} & \multirow{2}{*}{10} & EW & 0.136 & \multirow{2}{*}{ medium } & $50 \%$ & 0.43 & \multirow{2}{*}{1.8} \\
\hline & & & & & & NS & 0.041 & & MCE & 0.13 & \\
\hline \multirow{2}{*}{ EQ11 } & \multirow{2}{*}{$\begin{array}{l}\text { Loma } \\
\text { Prieta }\end{array}$} & \multirow{2}{*}{1989} & \multirow{2}{*}{ Gilroy } & \multirow{2}{*}{7.0} & \multirow{2}{*}{12} & EW & 0.17 & \multirow{2}{*}{ medium } & $90 \%$ & 0.42 & \multirow{2}{*}{4.7} \\
\hline & & & & & & NS & 0.16 & & MCE & 0.41 & \\
\hline \multirow{2}{*}{ EQ40 } & \multirow{2}{*}{$\begin{array}{c}\text { Palos } \\
\text { Verdes }\end{array}$} & \multirow{2}{*}{-} & \multirow{2}{*}{ Simulated } & \multirow{2}{*}{7.1} & \multirow{2}{*}{1.5} & EW & 1.37 & \multirow{2}{*}{ near } & $90 \%$ & 0.25 & \multirow{2}{*}{2.1} \\
\hline & & & & & & NS & 0.69 & & DBE & 0.22 & \\
\hline \multirow{2}{*}{ EQ30 } & \multirow{2}{*}{ Tabas } & \multirow{2}{*}{1974} & \multirow[t]{2}{*}{ - } & \multirow{2}{*}{7.4} & 12 & EW & 0.56 & near & $50 \%$ & 0.42 & 28 \\
\hline & & & & & 1.2 & NS & 0.50 & near & MCE & 0.39 & 2.0 \\
\hline EQ24 & Loma & 1080 & & & & EW & 1.31 & & $90 \%$ & 0.42 & \\
\hline & Prieta & 1989 & - & 7.0 & 3.5 & NS & 0.55 & near & MCE & 0.18 & 3.8 \\
\hline
\end{tabular}

${ }^{1}$ Moment Magnitudes, ${ }^{2}$ Closest Distances to Fault Rupture, ${ }^{3}$ Unadjusted $\mathrm{S}_{\mathrm{A}},{ }^{4}$ Scaled $\mathrm{S}_{\mathrm{A}}$. Source: SAC Earthquake Ground Motions, http://nisee.berkeley.edu/data/strong_motion/sacsteel/ground_motions.html 


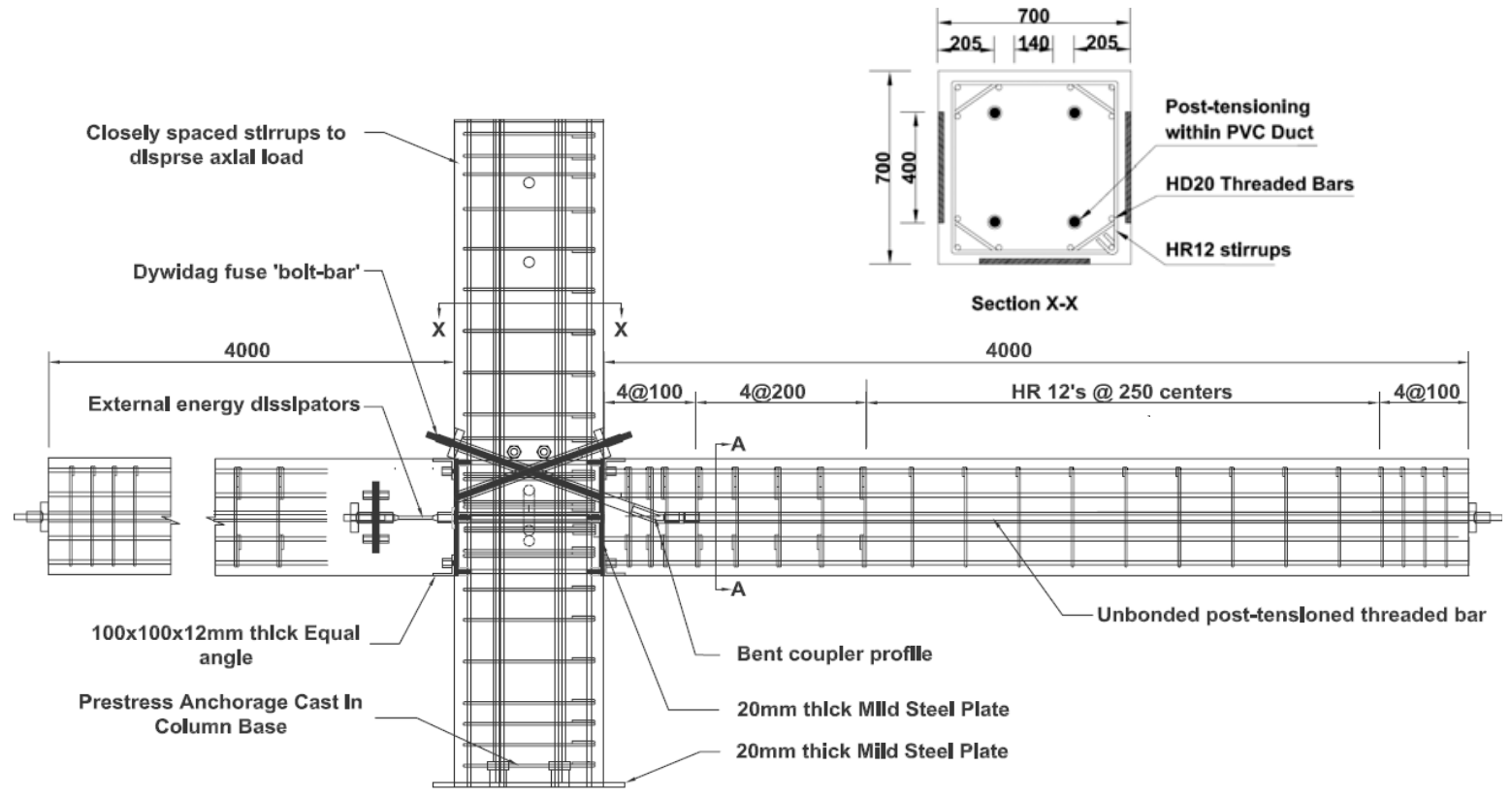

(a) Front (east-west) view of the test sub-assembly.
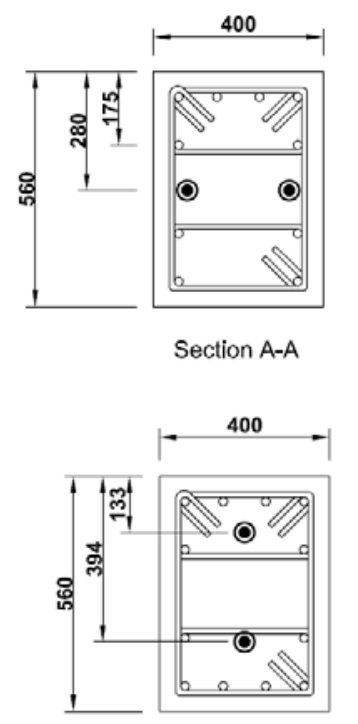

Section B-B

(b) Beam details

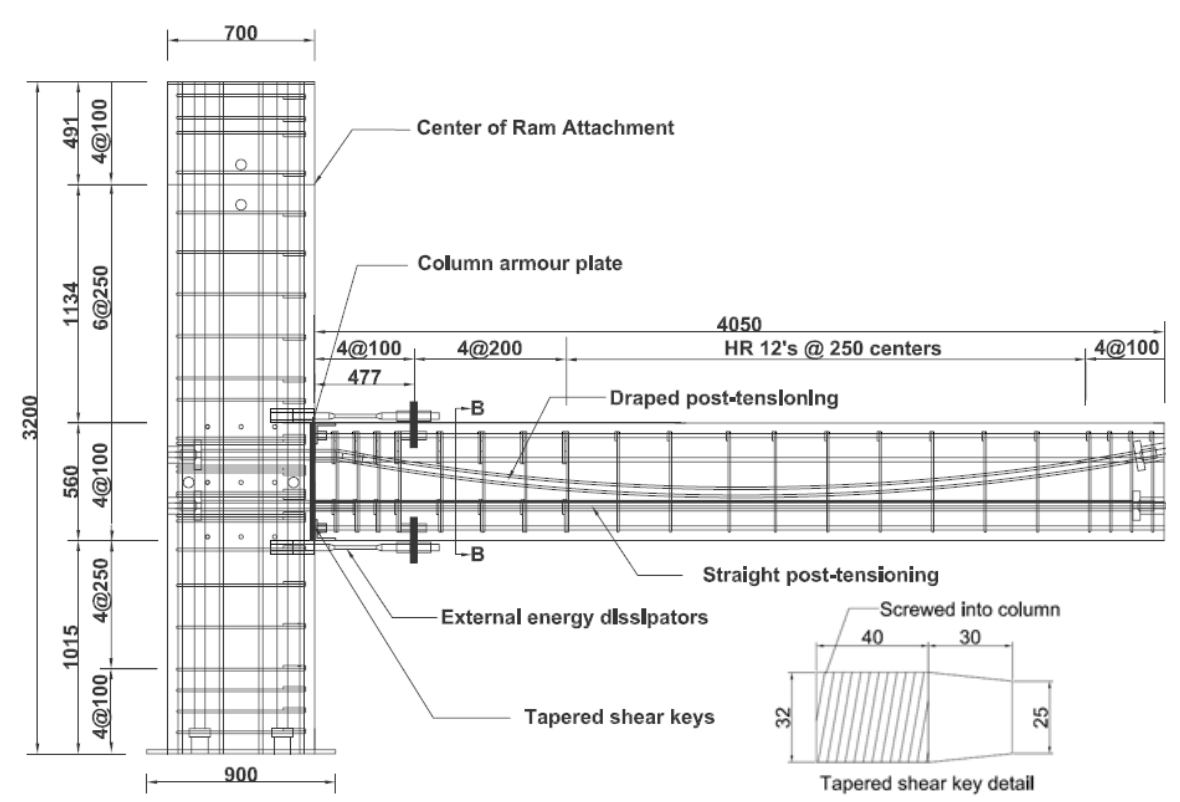

(c) Side (north-south) view of the test sub-assembly

Figure 1: Prototype building structure and reinforcement details of sub-assembly. 


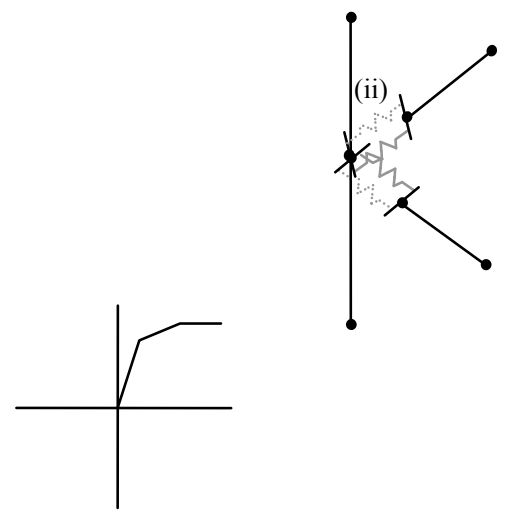

(i) Tri-linear elastic spring (post-tensioning

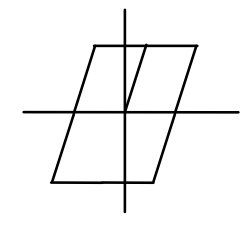

(ii) Elasto-plastic spring (energy dissipators

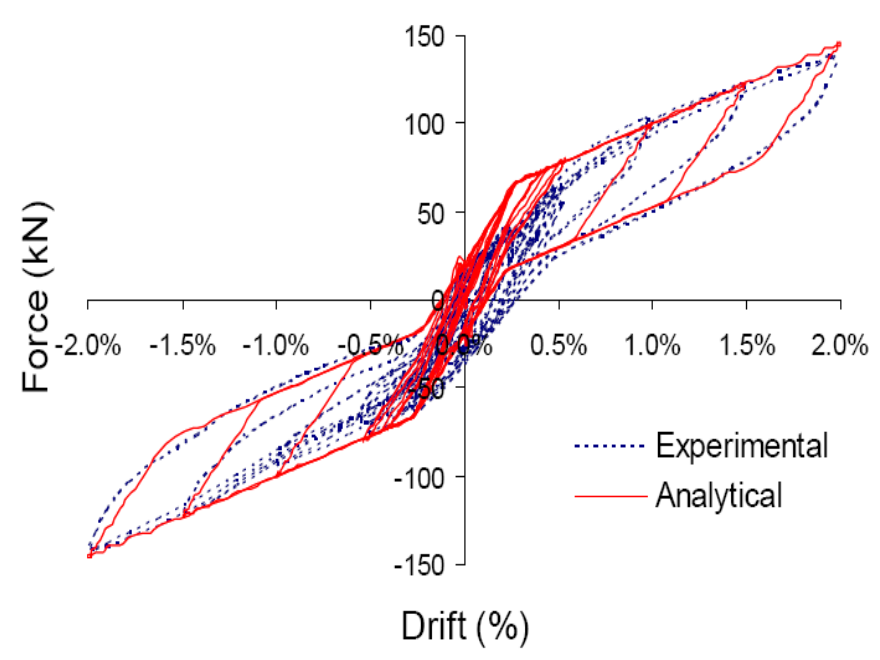

(b) Computational - experimental comparison

(a) Computational modelling

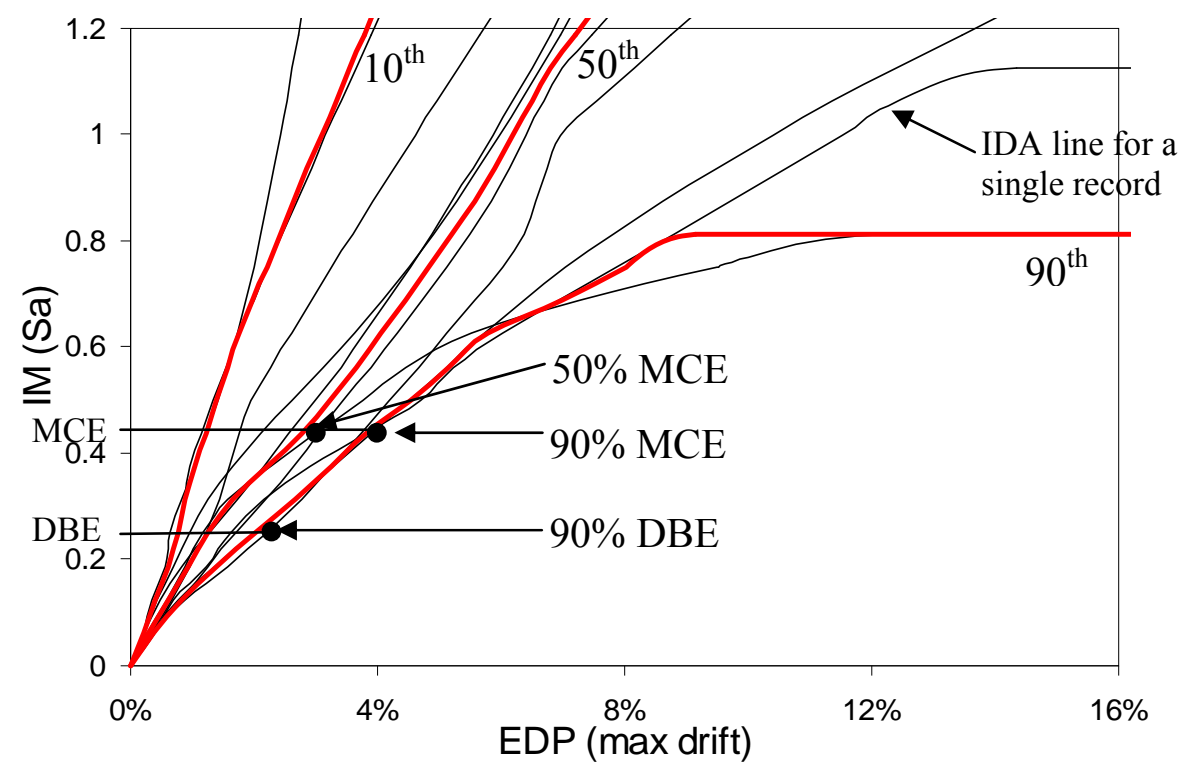

(c) Incremental dynamic analysis curves for near source suite

Figure 2: Loading protocol. 


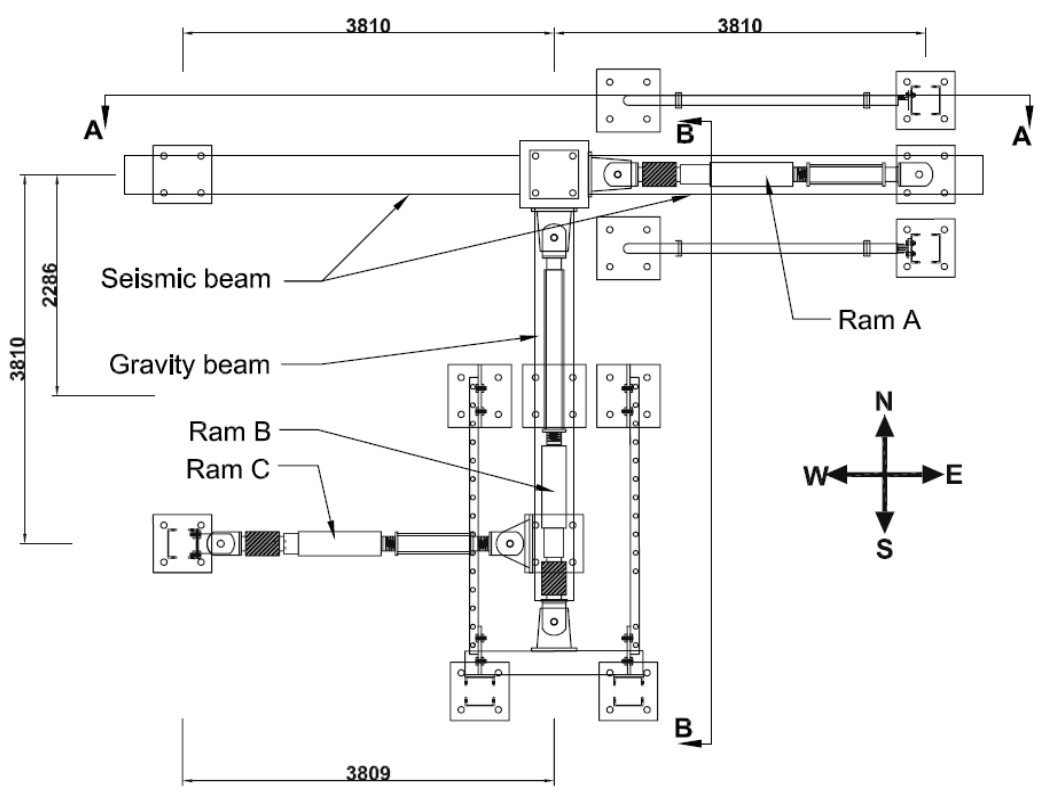

(a) Plan view

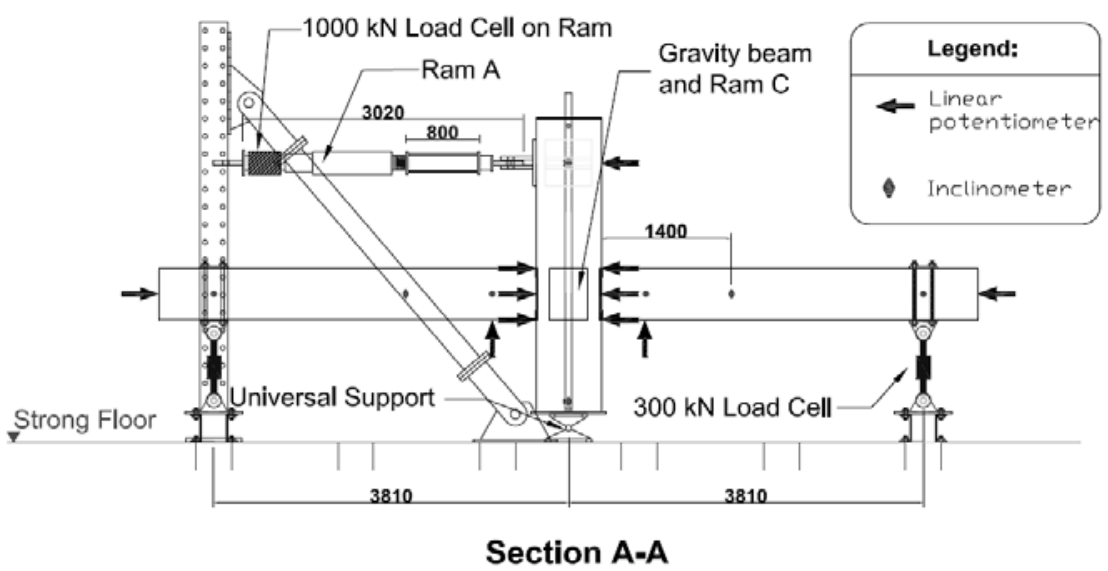

(b) Front elevation

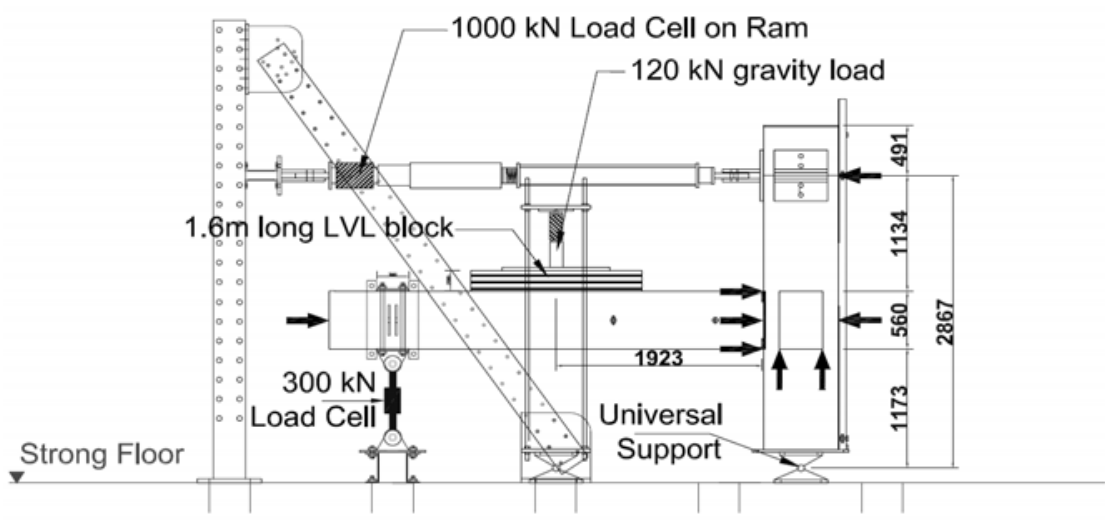

Section B-B

(c) End elevation

Figure 3: Experimental setup 


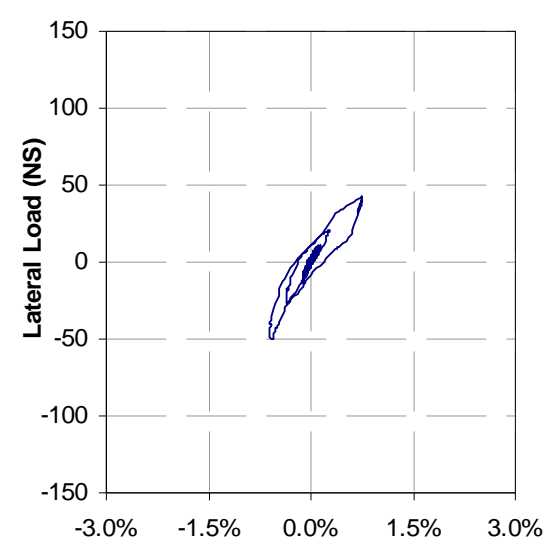

(a) N-S hysteretic response

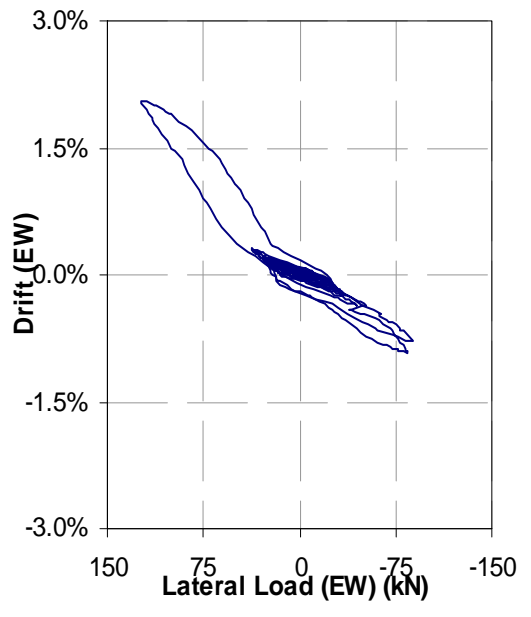

(b) E-W hysteretic response

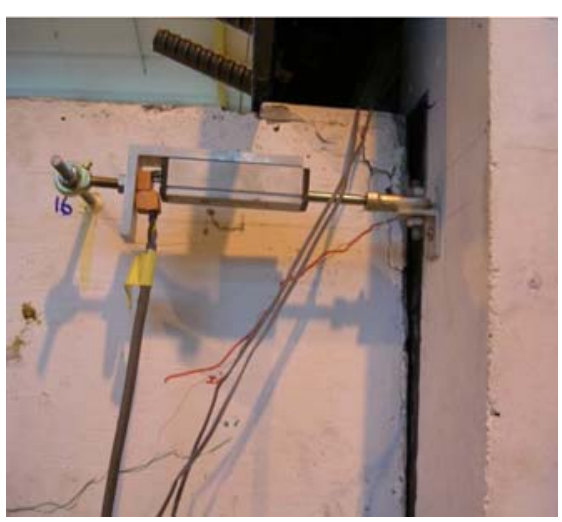

(e) Top of connection at $2 \%$ drift

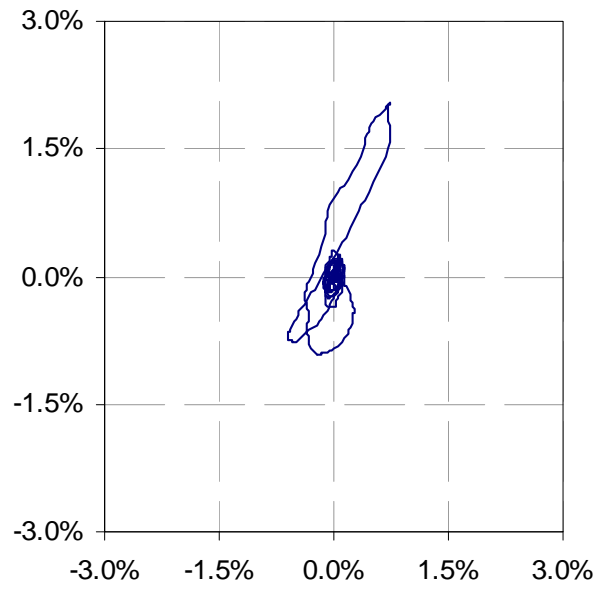

(c) $2 \mathrm{D}$ orbit of column

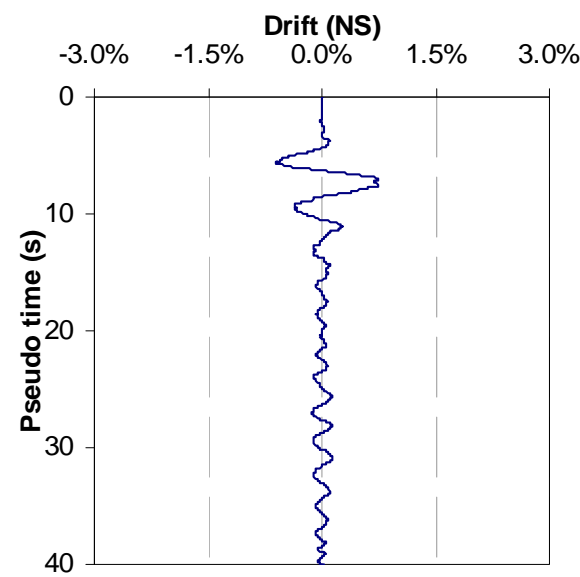

(f) N-S displacement time history

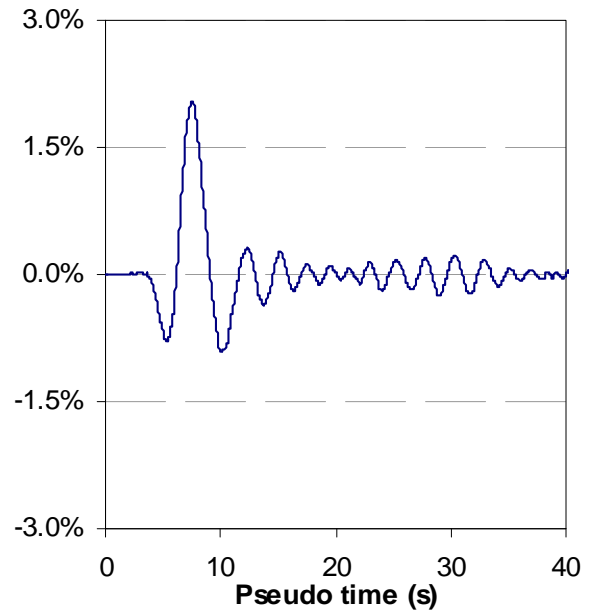

(d) E-W displacement time history

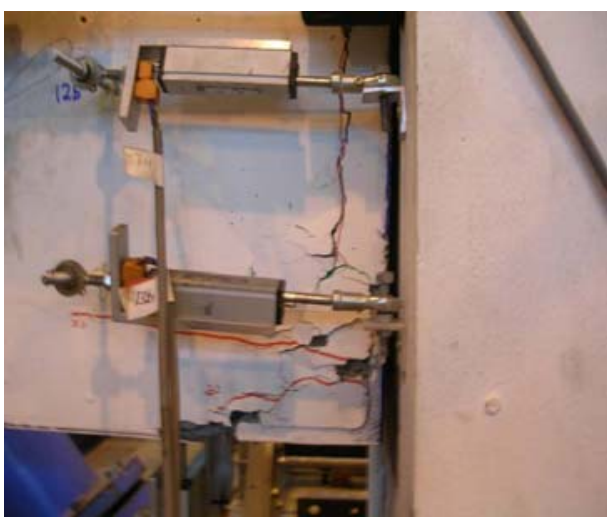

(g) Bottom of connection at $2 \%$ drift

Figure 4: Experimental results for the 90\% DBE near source ground motion (EQ40). 


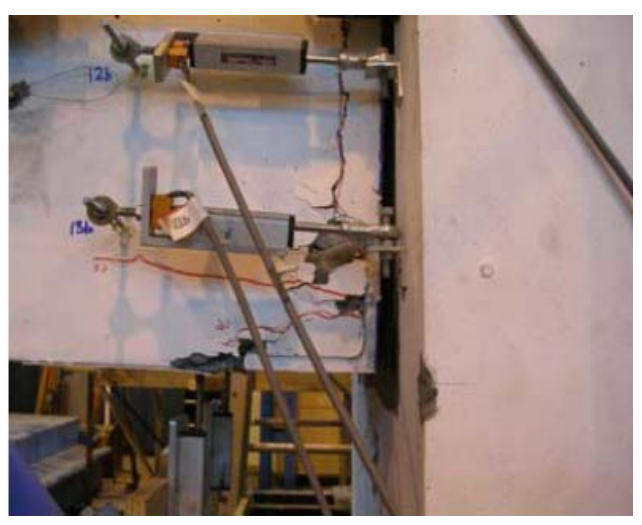

(a) Cover spalling at seismic beam connection

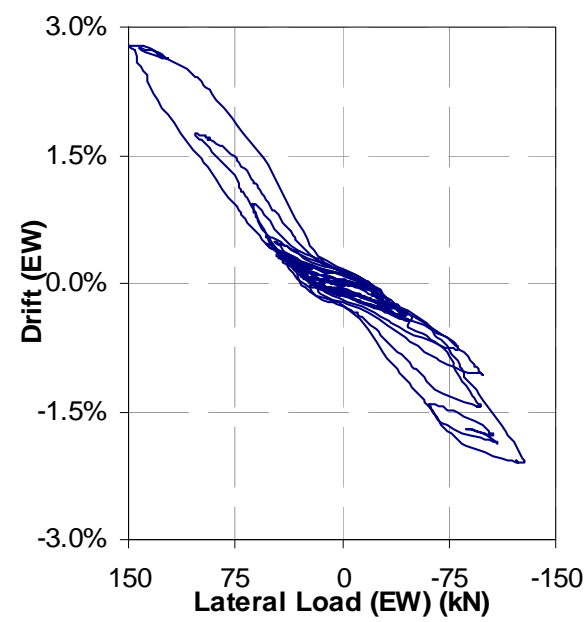

(d) E-W hysteretic response

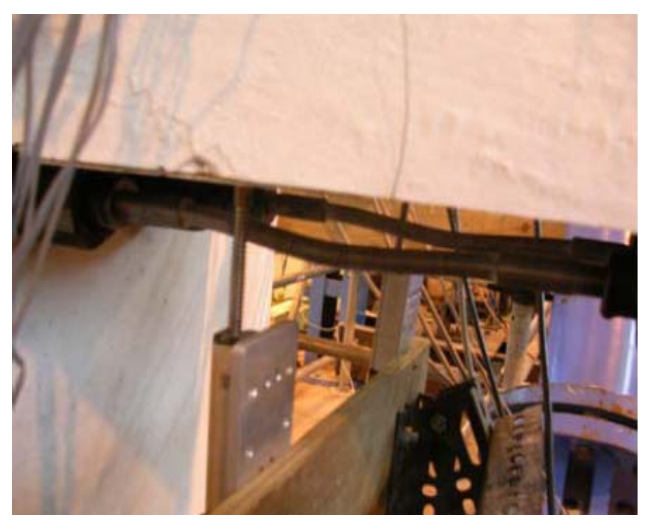

(g) Buckled gravity beam dissipators

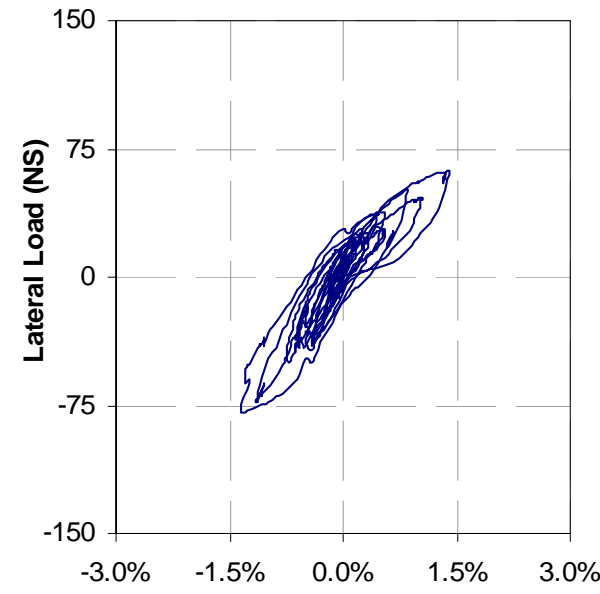

(b) N-S hysteretic response

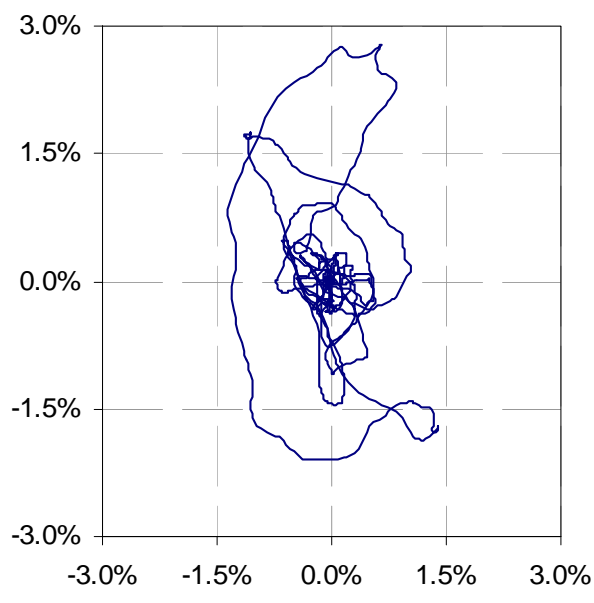

(e) 2D orbit of column

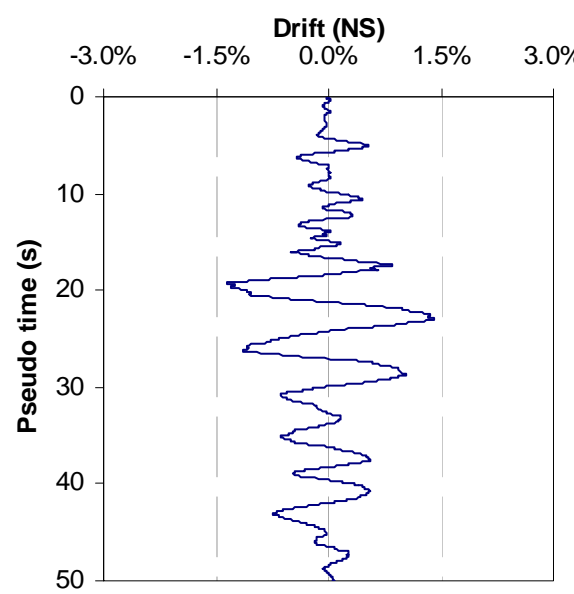

(h) N-S displacement time history

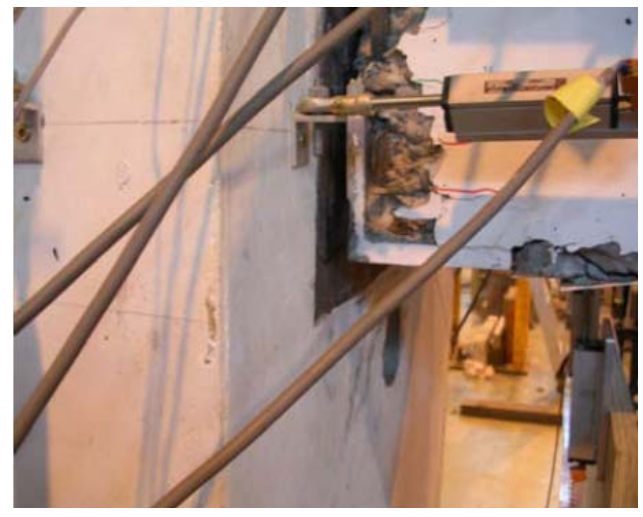

(c) Cover spalling at gravity beam connection

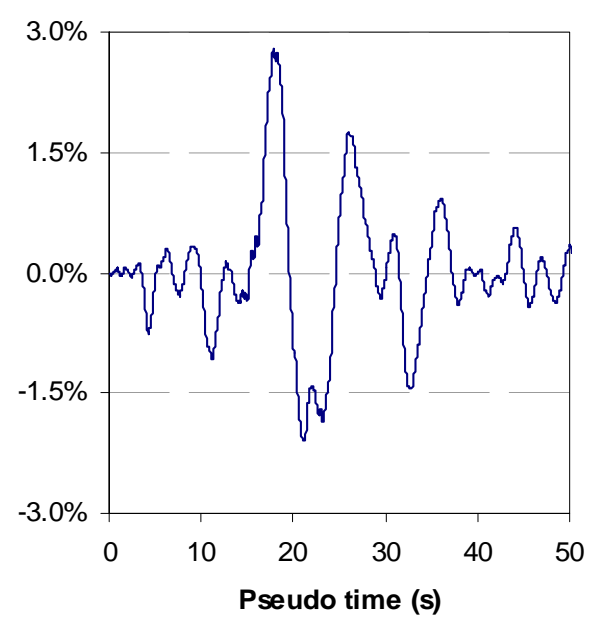

(f) E-W displacement time history

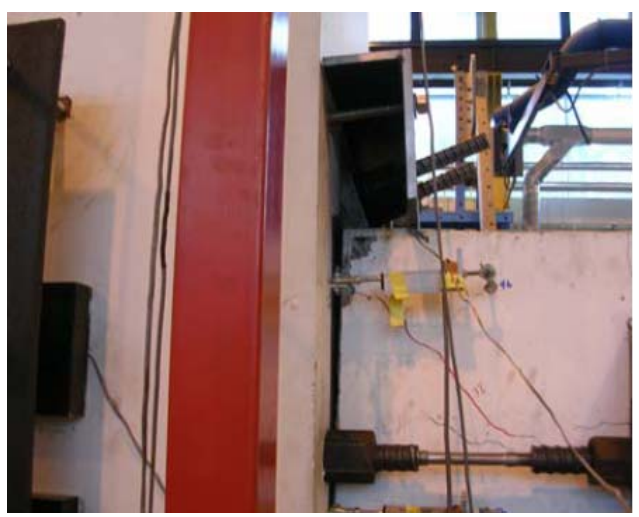

(i) Connection at $1.5 \% \mathrm{drift}$

Figure 5: Experimental Results for the 50\% MCE near source ground motion (EQ30). 


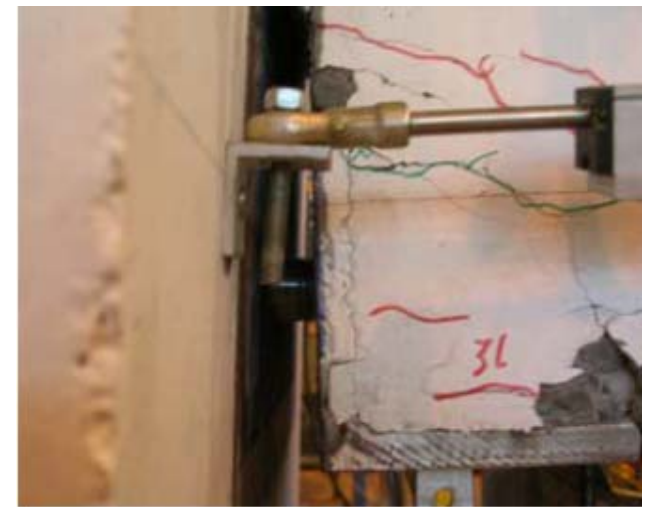

(a) Exposed shear key

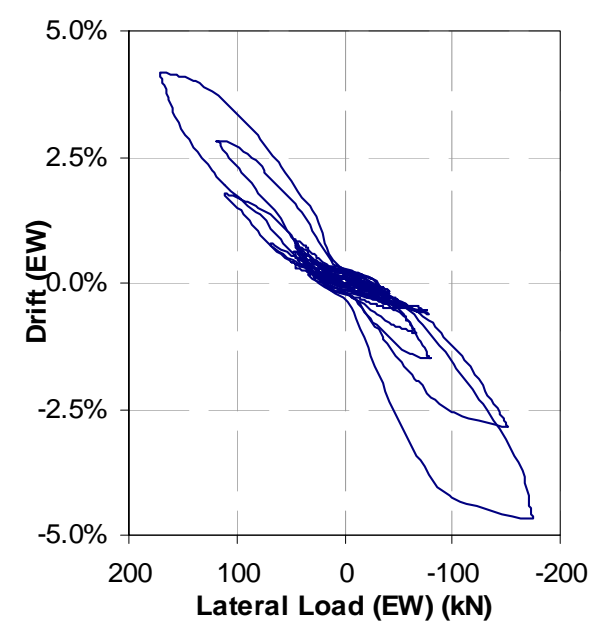

(d) E-W hysteretic response

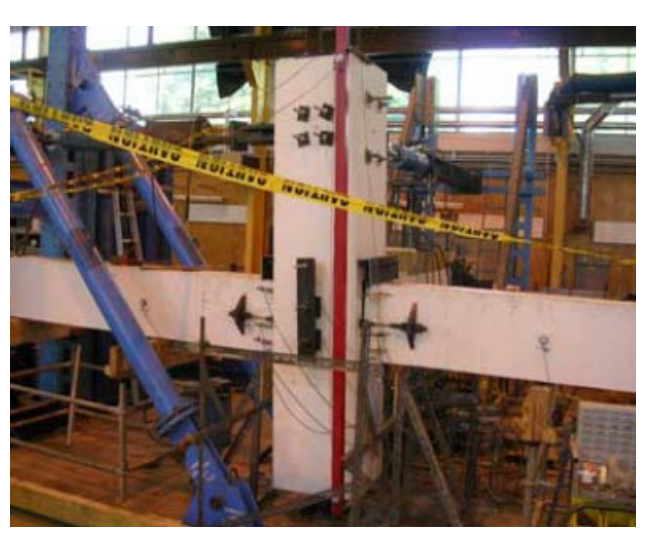

(g) Sub-assembly elevation

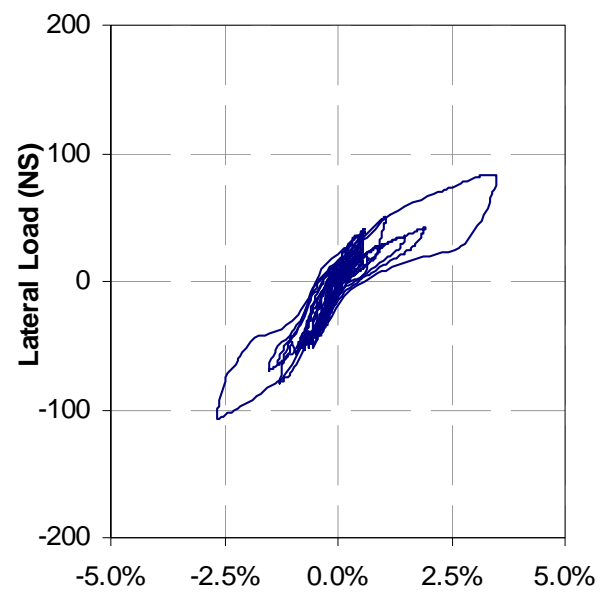

(b)-N-S hysteretic response

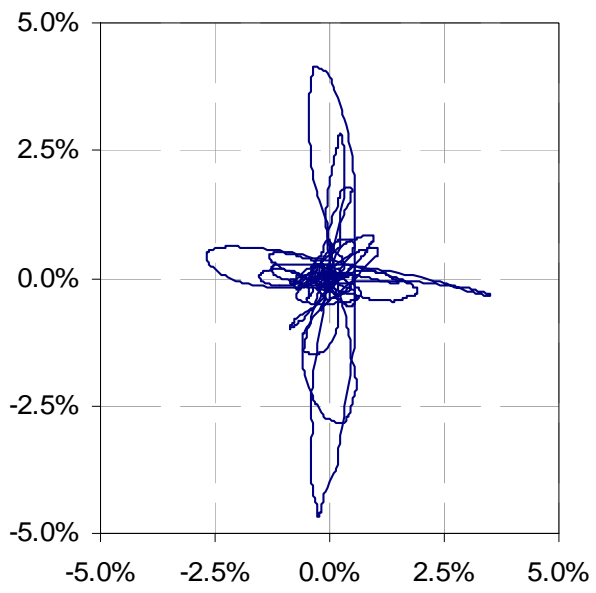

(e) $2 \mathrm{D}$ orbit of column

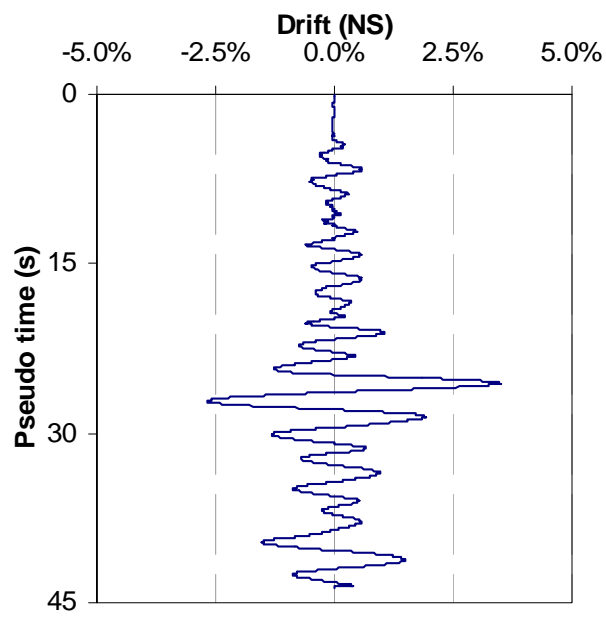

(h) N-S displacement time history

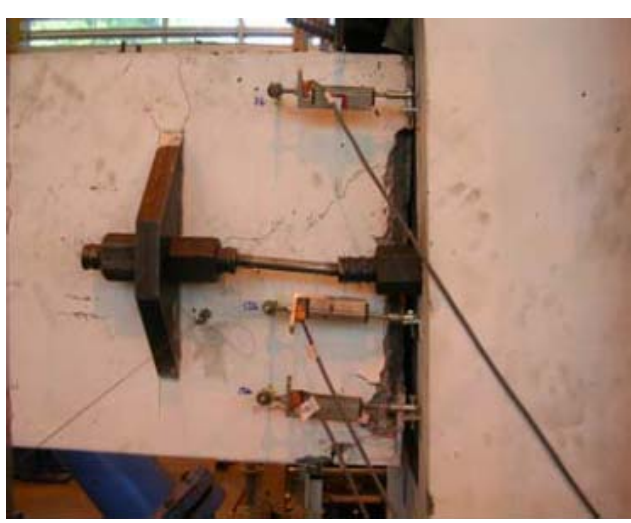

(c) Gap-opening at $4.7 \%$ drift

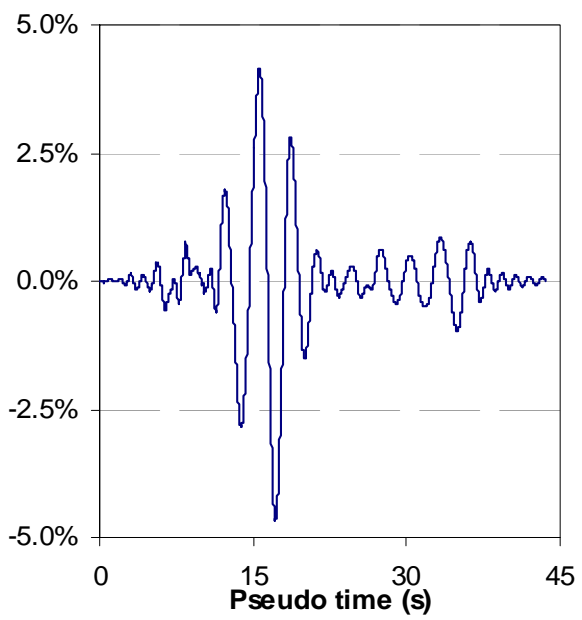

(f) E-W displacement time history

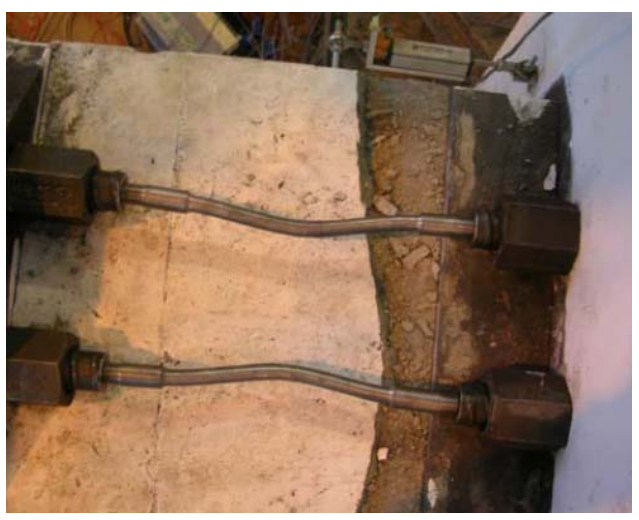

(i) Top view of buckled dissipators after re-centering

Figure 6: Experimental Results for the 90\% MCE medium source ground motion (EQ11). 